\title{
Vigilancia tecnológica e inteligencia competitiva para el sector funerario, caso "grupo Obelisco Ltda"
}

\section{Technological surveillance and competitive intelligence for the funeral sector, case "grupo Obelisco Ltda."}

\section{Resumen}

Este artículo muestra los resultados del proyecto: estudio de vigilancia tecnológica e inteligencia competitiva para la empresa Grupo Obelisco Ltda., orientado a la diversificación de su portafolio de servicios en la implementación del proceso de destino final de los restos humanos. El trabajo se adelantó bajo el enfoque mixto, cualitativo y cuantitativo, a través de un estudio descriptivo, tomando como base fundamental la metodología de vigilancia tecnológica Innovitech. A través de la búsqueda de información especializada, se pudo identificar una limitada producción científica frente a las tecnologías emergentes en disposición de restos humanos, convirtiéndose en un tema poco documentado. Teniendo en cuenta las características de la empresa Grupo Obelisco Ltda., las tendencias del sector y el objetivo de la investigación, se concluye que dentro de las tendencias tecnológicas más viables son: urna biodegradable, proceso de hidrólisis alcalina, Criodesecación y Cementerio ecológico.

Palabras clave: vigilancia tecnológica, inteligencia competitiva, sector funerario, destino final.

\section{Rocío del Mar Rodríguez-Parra' \\ Yolanda González-Castro}

Recibido: agosto 21 de 2018 Aceptado: octubre 02 de 2018

\begin{abstract}
This article shows the results obtained in the study called "Study of technological surveillance and competitive intelligence for the company Grupo Obelisco Ltda, which was oriented to the diversification of its service portfolio in the implementation of the final destination process. The described project was developed under the mixed (qualitative and quantitative) approach, through a descriptive study taking as a fundamental basis of its development the technological surveillance methodology called Innovitech. Through a specialized research, a limited scientific production could be identified in the face of emerging technologies in the disposal of human remains, becoming a little documented subject. Taking into account the characteristics of the company Grupo Obelisco Ltda., the trends of the sector and the research objective, it is concluded that within the technological trends the most viable are: biodegradable urn, alkaline hydrolysis process, cryo-drying and ecological cemetery.
\end{abstract}

Keywords: technological surveillance, competitive intelligence, funerary sector, final destination.

1 Administradora de Empresas, Magíster en Administración de Organizaciones, Profesional de Planeación del Centro de Gestión Administrativa y Fortalecimiento Empresarial, Tunja, Colombia. E-mail: rocimar129@yahoo.com

2 Administradora de Empresas, Doctora en Administración de Negocios, Universidad Nacional Abierta y a Distancia, Pamplona, Colombia.E-mail: yolanda.gonzalez@unad.edu.co 


\section{Introducción}

En los últimos años, en el ámbito nacional, el sector funerario ha alcanzado un crecimiento del 9\% anual, con ingresos de $\$ 738.000$ millones por año, generando más de 30.000 empleos directos. La participación del sector en el PIB de Colombia ha sido del $0,12 \%$, donde el servicio de previsión exequial genera $\$ 353.000$ millones, servicio al que están afiliadas o inscritas unos 17 millones de personas, aproximadamente. Este crecimiento ha permitido que Colombia se ubique en el tercer puesto, después de Estados Unidos y España, proyectando un sector reinventado e innovador (Revista Dinero, 2016).

El Grupo Obelisco Ltda., es una empresa boyacense de más de 10 años de experiencia en el mercado de la previsión exequial; está conformada por 10 funerarias del departamento de Boyacá, lo que le ha permitido ganar un alto reconocimiento y obtener una participación significativa con un $30 \%$ del mercado de previsión exequial del departamento. La empresa cuenta con un aproximado de 20 mil contratantes y ha prestado 3.000 servicios funerarios desde su creación, cubriendo tres servicios estipulados en su contrato de previsión: a) servicio funerario, b) traslado de la persona fallecida al lugar de residencia habitual, a nivel regional, nacional según sea el caso; c) destino final, ya sea inhumación o cremación en parques cementerios distritales o privados.

En cuanto al destino final que realiza el grupo Obelisco Ltda, La inhumación se realiza disponiendo los restos humanos en bóvedas o lotes que se arriendan de 4 a 6 años, utilizando cofres o ataúdes en madera; la cremación del cuerpo humano se realiza en hornos crematorios y el procedimiento dura entre una y cinco horas (Recondo, 2013).

Los dos tipos de servicios plantean problemáticas de tipo social, ambiental y económico, que inicia desde el proceso de embalsamamiento del cuerpo para que se conserve para las ceremonias funerarias, momento de la última despedida. De acuerdo con Quintero (2017) los líquidos empleados para esta práctica poseen componentes como el arsénico, el mercurio y el formaldehído teniendo un profundo efecto contaminante en el agua y considerados cancerígenos por la Organización Mundial de la Salud, OMS.

Específicamente, en el caso de la inhumación, el impacto económico que se genera para la familia va desde el pago continuo del arrendamiento del lote o bóveda hasta la compra de los ataúdes o cofres. Frente al impacto ambiental, se destaca que para la fabricación de un ataúd se requiere talar un árbol, lo cual genera que haya 7,3 millones de hectáreas de árboles menos, por año en el mundo (Velasco, Zea y Marlevis, 2012); esto además de generar emisión de dioxinas y monóxido de carbono a la atmósfera, provocadas por la descomposición del cuerpo en el ataúd, lo que se prolonga durante al menos quince años.

Con respecto a la cremación, se identifica que el cuerpo cremado emite gases de combustión que serán canalizados a la atmósfera, generando compuestos como el vapor de agua, monóxido de carbono y dióxido de azufre, contribuyendo a una porción muy pequeña de los gases de efecto invernadero; esto además de liberar entre 0,8 y 5,9 gr de mercurio, donde el $75 \%$ de este material se dirige al aire, mientras que el resto se conduce al suelo y al agua, generando daños a nivel del sistema nervioso central del ser humano (Gaioli, Amoedo \& González, 2012).

Frente a estos efectos, no hay una alternativa ecológica en Boyacá, siendo su uso continuo y extendido una problemática de orden ambiental, social y económico para las comunidades y las familias que reciben el servicio funerario (Cepeda, comunicación personal, 04 de septiembre de 2017). Esto genera en la empresa bajo estudio, la necesidad de diversificar su portafolio con una alternativa de destino final que minimice la problemática descrita y cuyo mantenimiento y sostenibilidad sea adecuada para la organización. 
En tal sentido, es pertinente recordar lo que refieren Escandón, Rodríguez y Hernández (2013), citados por Rodríguez (2018), en cuanto a que la dinámica del mundo actual genera en las empresas la necesidad de buscar nuevas estrategias que les permitan mantenerse y crecer dentro del mercado. Por ello surge la gestión del conocimiento como una necesidad para toda organización, junto con la vigilancia tecnológica, como una herramienta fundamental para alcanzar los objetivos de mejoramiento organizacional, permanencia y diferenciación en el mercado.

En esta dirección y resaltando la importancia y propósito de la empresa Obelisco Ltda, de asumir un rol activo de desarrollo e innovación que permita un crecimiento empresarial, sectorial, y económico en su entorno, se planteó como pregunta de investigación: ¿Qué tendencias tecnológicas, amigables con el medio ambiente y sostenibles económicamente frente al servicio de destino final, pueden ser implementadas por la empresa Grupo Obelisco Ltda. para diversificar su portafolio de servicios e innovar en su cadena de valor?.

\section{Marco teórico y métodos}

\subsection{Fundamentación teórica}

\section{Gestión del conocimiento}

La Gestión del Conocimiento, GC, es un proceso que implica desarrollar, estructurar, mantener $y$ transformar la información en un activo crítico y ponerla a disposición de unos usuarios, para obtener mejores resultados en el desarrollo de sus actividades (Aja, 2002). En este sentido, la GC se ha convertido en uno de los principales temas de investigación y en el paradigma de gestión por excelencia de las empresas (Ahumada, Zarate Corne- jo, Plascencia \& Perusquia, 2012) convirtiendo a las empresas que gestionen esta disciplina en organizaciones inteligentes, capaces de responder a las exigencias del mercado (Rodríguez, 2006).

En este escenario, la creación de conocimiento organizacional se convierte en la clave de las empresas para innovar y generar ventajas competitivas (Nonaka, Takeuchi \& Kocka, 1999). Por esta razón, las empresas deben desarrollar la capacidad de generar nuevos conocimientos, transmitirlos entre los miembros de la organización y materializarlos en productos, servicios y sistemas.

\section{Vigilancia tecnológica}

Marulanda, Hernández y López (2016), afirman que a partir del modelo de las cinco fuerzas de Porter (1979) se definen cuatro tipos de vigilancia según el fin que se desea lograr: (a) competitiva, (b) comercial, (c) del entorno, (d) tecnológica. Esta última, se considera como un proceso organizado, selectivo y permanente, de captar información del exterior y de la propia organización sobre ciencia y tecnología, para: seleccionarla, analizarla, difundirla, comunicarla y convertirla en conocimiento, para tomar decisiones con menor riesgo y poder anticiparse a los cambios (González, Gómez \& Muñoz, 2015).

Dicho esto, Montes (2014), citado por San Juan y Romero (2016), relaciona los modelos de vigilancia tecnológica a partir de dos enfoques: (a) modelos para implementar y estructurar los sistemas de vigilancia tecnológica; (b) modelos para desarrollar operativamente la vigilancia tecnológica, algunos de los cuales se listan en la tabla 1. En El desarrollo del proyecto se consideró el uso de la metodología Innovitech, que será abordada en el marco metodológico. 
Tabla 1. Clasificación de los modelos de vigilancia tecnológica, de acuerdo a su enfoque.

\begin{tabular}{lll}
\hline Modelos & $\begin{array}{l}\text { Modelos para implementar y es- } \\
\text { tructurar }\end{array}$ & $\begin{array}{l}\text { Modelos para desarrollo opera- } \\
\text { tivo de la V.T. }\end{array}$ \\
\hline Salgado, Guzmán y & $\mathrm{X}$ & $\mathrm{X}$ \\
Carrillo (2003) & & $\mathrm{X}$ \\
Castro (2007) & $\mathrm{X}$ \\
Malaver y Vargas (2007) & \\
Colciencias - TRIZ XXI (2006) & $\mathrm{X}$ \\
Coca, García, Santos y Fernández (2010) & $\mathrm{X}$ & \\
García, Ortoll y López (2011) & & $\mathrm{X}$ \\
Oroz (2013) & $\mathrm{X}$ & \\
Montes (2014) & $\mathrm{X}$ & \\
Innnovitech (2015) & & \\
\hline
\end{tabular}

\subsection{Metodología}

El abordaje de la presente investigación se realizó desde un enfoque mixto; cualitativo tipo análisis documental y cuantitativo no experimental transversal, a través de un estudio exploratorio y descriptivo, a partir de la metodología Innovitech perteneciente al modelo de Vigilancia Tecnológi$\mathrm{ca}, \mathrm{VT}$.
La metodología Innovitech, establecida por González, Gómez, y Muñoz (2015), determina que la VT se desarrolla en función de la toma de decisiones, previo al desarrollo de unas fases cíclicas, que permiten la realimentación en cualquier momento, de acuerdo a lo resultados obtenidos en cada etapa, y existe una integración entre el público objetivo y las áreas vigiladas. Este modelo se compone de siete fases, desarrolladas como se describe en la tabla 2.

Tabla 2. Fases del modelo Innovitech.

\begin{tabular}{|c|c|c|}
\hline Fase & Objetivo & Desarrollo \\
\hline $\begin{array}{l}\text { 1. Identificación } \\
\text { de necesidades }\end{array}$ & $\begin{array}{l}\text { Identificar las problemáticas } \\
\text { o necesidades de la empresa } \\
\text { Grupo Obelisco }\end{array}$ & $\begin{array}{l}\text { Se realizó una entrevista estructurada, compuesta de veintitrés } \\
\text { preguntas estimadas en la metodología innnovitech, con opción } \\
\text { abierta al gerente de la empresa. }\end{array}$ \\
\hline 2. Diagnóstico & $\begin{array}{l}\text { Identificar el estado actual de } \\
\text { los principales componentes } \\
\text { de la VT }\end{array}$ & $\begin{array}{l}\text { Se analizó: generalidades de la empresa, fuentes de ingreso, por- } \\
\text { tafolio de servicios, investigación, desarrollo e innovación (I+D+i), } \\
\text { clientes, financiero, competencia y productos sustitutos, provee- } \\
\text { dores; estas categorías fueron valoradas bajo las calificaciones } \\
\text { cualitativas: bajo, básico, alto, superior }\end{array}$ \\
\hline $\begin{array}{l}\text { 3. Definición del } \\
\text { factor crítico a } \\
\text { vigilar }\end{array}$ & $\begin{array}{l}\text { Definir las palabras o conjun- } \\
\text { to de palabras que atiende la } \\
\text { necesidad de la organización }\end{array}$ & $\begin{array}{l}\text { Teniendo los resultados del diagnóstico realizado y lo expuesto } \\
\text { en la introducción, se definen dos factores críticos a vigilar: (1) } \\
\text { innovación tecnológica en las prácticas funerarias, (2) alternativas } \\
\text { de destino final. }\end{array}$ \\
\hline
\end{tabular}




\begin{tabular}{|c|c|c|}
\hline Fase & Objetivo & Desarrollo \\
\hline $\begin{array}{l}\text { 4. Búsqueda y } \\
\text { recolección de } \\
\text { Información }\end{array}$ & $\begin{array}{l}\text { Establecer las fuentes de in- } \\
\text { formación y acceder a ellas } \\
\text { para realizar la captación de } \\
\text { lo que es requerido }\end{array}$ & $\begin{array}{l}\text { Se realizó la búsqueda en: documentos de la empresa, bases } \\
\text { de datos, patentes, entre otras. Las palabras clave usadas en la } \\
\text { búsqueda de información, fueron: innovación, tecnología, sec- } \\
\text { tor funerario, inhumación, cremación. La información se recopiló } \\
\text { en una bitácora compuesta por: ecuación de búsqueda, base de } \\
\text { datos, fecha de la búsqueda, rango de años, si es pertinente esa } \\
\text { ecuación, número de resultados, entre otros. }\end{array}$ \\
\hline 5. Análisis & $\begin{array}{l}\text { Seleccionar los resultados } \\
\text { relevantes y aplicar herra- } \\
\text { mientas estadísticas para } \\
\text { identificar zonas geográficas, } \\
\text { autores, u otros. }\end{array}$ & $\begin{array}{l}\text { Se seleccionaron los resultados pertinentes al factor crítico a vi- } \\
\text { gilar, tanto en producción científico-académica como patentes; } \\
\text { se realizó un mapa tecnológico con análisis de frecuencias y coo- } \\
\text { currencia, identificando la tendencia de producción científica a } \\
\text { través de las palabras clave seleccionadas. }\end{array}$ \\
\hline $\begin{array}{l}\text { 6. Elaboración } \\
\text { de informes }\end{array}$ & $\begin{array}{l}\text { Plasmar en un informe la in- } \\
\text { formación relevante luego } \\
\text { del proceso de búsqueda y } \\
\text { análisis. }\end{array}$ & $\begin{array}{l}\text { Se presentó un informe a la empresa a través de una cartilla, y } \\
\text { se hizo la socialización de hallazgos para la respectiva toma de } \\
\text { decisiones }\end{array}$ \\
\hline $\begin{array}{l}\text { 7. Difusión o } \\
\text { Modelo }\end{array}$ & $\begin{array}{l}\text { El objetivo de esta etapa es } \\
\text { que la información analizada } \\
\text { llegue a los decisores de una } \\
\text { manera práctica }\end{array}$ & \\
\hline
\end{tabular}

\section{Resultados y discusión}

\subsection{Búsqueda en bases de datos}

En la figura 1 se relaciona el número de resultados arrojados por cada base de datos, teniendo en cuenta: las palabras clave, factor crítico de vigilancia y las siguientes ecuaciones de búsqueda: Innovación AND tecnología AND"sector funerario, funeraria OR funerario AND cremación OR inhumación AND tecnología, que fueron empleadas en la búsqueda de información.

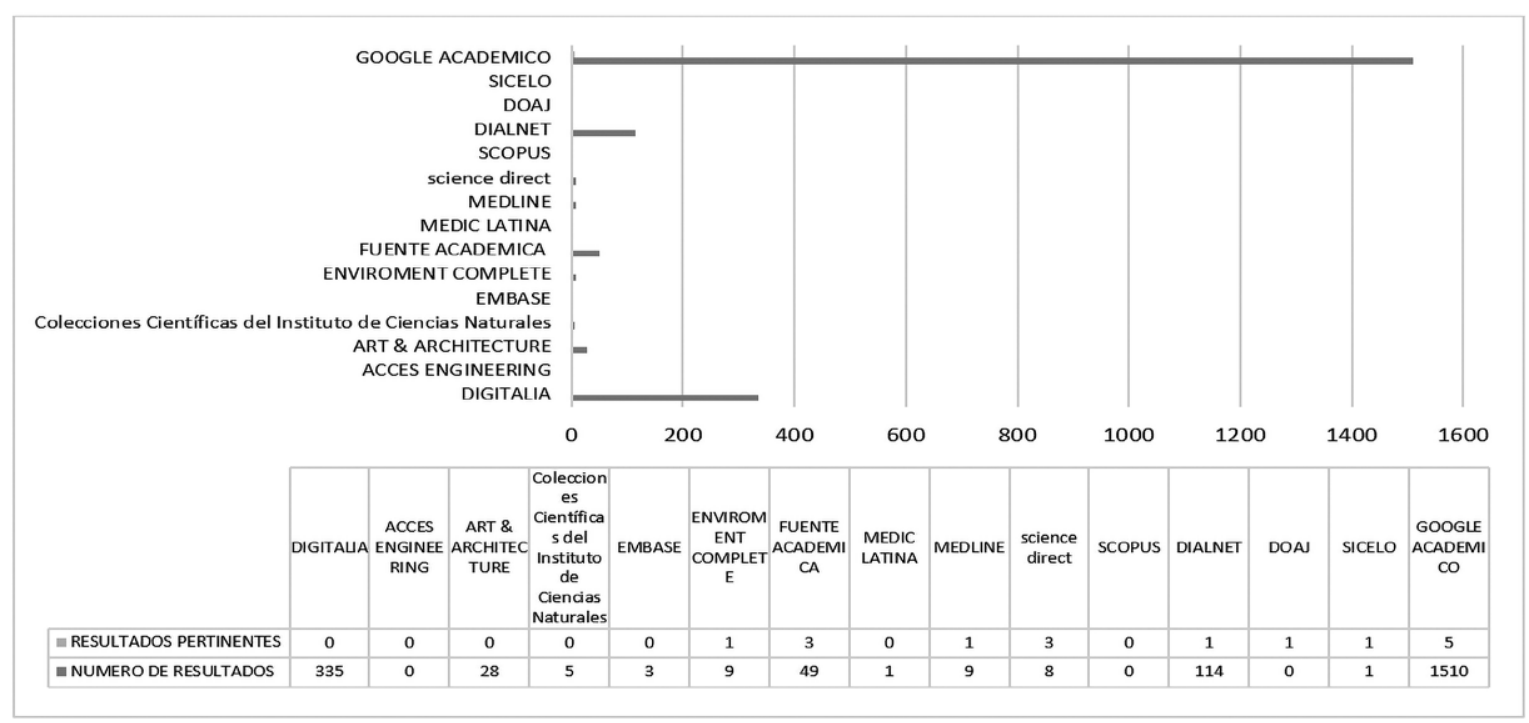

Figura 1. Comportamiento de la búsqueda en bases de datos. 
Para establecer un contexto del estado de la investigación del objeto de estudio, se relaciona la información disponible sobre investigaciones formales a partir de los datos encontrados en las bases de datos especializadas, ver tabla 3, dando prevalencia a aquellas que tuvieron resultados pertinentes en la búsqueda realizada.

Tabla 3. Resultados de Búsqueda en Bases de Datos Especializadas.

\begin{tabular}{|c|c|c|c|}
\hline $\begin{array}{c}\text { Base de } \\
\text { datos }\end{array}$ & Resultados & $\begin{array}{l}\text { Resultado } \\
\text { pertinente }\end{array}$ & Artículo pertinente \\
\hline $\begin{array}{l}\text { Enviroment } \\
\text { Complete }\end{array}$ & 9 & 1 & Burial grounds' impact on groundwater and public health: an overview \\
\hline $\begin{array}{l}\text { Fuente Aca- } \\
\text { démica }\end{array}$ & 49 & 3 & $\begin{array}{l}\text { La ritualidad en transición. Un estudio sobre las preferencias del des- } \\
\text { tino corporal } \\
\text { Resting in Peace: The Work of Mourning in Six Feet Under } \\
\text { Mémora lidera la profesionalización del sector funerario }\end{array}$ \\
\hline Medline & 9 & 1 & Change and Innovation in the Funeral Industry \\
\hline $\begin{array}{l}\text { Science } \\
\text { rect }\end{array}$ & 3 & 2 & $\begin{array}{l}\text { Present and future emissions of HAPs from crematories in China } \\
\text { The potential dangers of medical devices with current cremation } \\
\text { Toxic emissions from crematories: a review }\end{array}$ \\
\hline Scopus & 114 & 1 & $\begin{array}{l}\text { Cemitérios, crematórios e novas tecnologias fúnebres: impactos am- } \\
\text { bientais e preferências post-mortem na cidade de Maceió - AL }\end{array}$ \\
\hline $\begin{array}{l}\text { Google Aca- } \\
\text { démico }\end{array}$ & 1510 & 5 & $\begin{array}{l}\text { Tecnologías alternas eco sustentables en el depósito de restos huma- } \\
\text { nos Panteón civil San Lorenzo Tezonco } \\
\text { Plan de negocios para una empresa productora y comercializadora de } \\
\text { ataúdes fúnebres metálicos con sede en la ciudad de Bogotá } \\
\text { Eliminación del cuerpo en Portugal; prácticas actuales y potenciales, } \\
\text { adopción de hidrólisis alcalina y entierro natural como alternativas sos- } \\
\text { tenibles } \\
\text { Estudio para el diseño del cementerio ecológico municipal para la pa- } \\
\text { rroquia urbana del cantón Milagro provincia del Guayas } \\
\text { Cementerios, crematorios y nuevas tecnologías funerales, Estudio de } \\
\text { viabilidad de un crematorio solar en la India }\end{array}$ \\
\hline
\end{tabular}

Los artículos y estudios identificados como pertinentes para el tema de estudio, fueron extraídos y resumidos para ampliar los conocimientos que se abordan en esta área. A continuación, se hace una breve reseña de la producción científica pertinente.

\section{Impacto ambiental y salubre frente al destino final}

Oliveira et al. (2013), describen una visión general de la amenaza potencial de los cementerios frente a impactos ambientales y riesgos para la salud pública, que de esta práctica emergen. Los autores señalan que se deben realizar estudios más com- pletos sobre: el proceso de descomposición, la influencia de los materiales usados en las prácticas funerarias, el uso de sustancias oxidantes, los procedimientos de embalsamamiento, entre otros aspectos, que permitan prevenir los riesgos ambientales y el impacto en la salud. Igualmente, indican las diferencias encontradas entre los 3 tipos de cementerios usados como opción de destino final.

Xue, et al. (2016), describen como la cantidad de población muerta en China ha llegado a 9.65 millones, donde el $49.5 \%$ de los cadáveres humanos es cremado en aproximadamente 1700 crematorios, diseminados por todo el país, según cifras del 2012. Esto genera una descarga considerable de 
diversos contaminantes atmosféricos peligrosos, ocasionando grandes preocupaciones sobre la calidad del aire regional y riesgos para la salud de los residentes de los alrededores. Los autores indican que para mitigar los riesgos causados por los crematorios, es necesario implementar estándares de emisión más estrictos, optimizando la combustión; lo cual requiere la instalación del mejor sistema de purificación de gases disponible.

Smith, Gitsham, Donell, Rose y Hing (2012), en su estudio refieren la indagación de problemas que rodean la cremación de dispositivos médicos en pacientes fallecidos, asociando la incidencia de los diferentes tipos de dispositivos incinerados con los peligros ambientales y de salud. Los resultados indicaron que los marcapasos y clavos ortopédicos, son dos peligros potenciales para el personal de cremación. Igualmente, existe confusión sobre los peligros potenciales de la exposición a radionucleídos, especialmente sobre el efecto del tiempo entre la introducción del material y la muerte, con respecto a su: manipulación, almacenamiento y cremación. Además, se evidencia que existen problemas de salud pública que rodean las emisiones de mercurio de la cremación de amalgamas dentales.

En el artículo de Mari y Domingo (2010), se indica como en los últimos años la proporción de cadáveres de cremación ha aumentado dramáticamente en muchos países; esto ha permitido identificar en los crematorios a fuentes de contaminantes ambientales, como: dibenzo-p-dioxinas policloradas y dibenzofuranos, PCDD / Fs, y mercurio, entre los que más preocupan. Los autores afirman que, a diferencia de otros procesos de incineración para los que el número de estudios sobre sus emisiones tóxicas es considerable, las referencias relacionadas con PCDD / F y las emisiones de mercurio de los crematorios y sus riesgos para la salud, son muy limitadas.

El trabajo de Da Cruz, Lezana, Dos Santos, Zancan y Pinto (2015), describe que los cementerios y crematorios son las principales formas funerarias usa- das hoy en día, además de ser un segmento poco estudiado en cuanto a sus impactos medioambientales. Los autores señalan que la mayoría de la población desconoce los impactos ambientales que generan estas prácticas; además, indican que son poco conocidos otros procesos que se están utilizando en el sector fúnebre, tales como: la liofilización y la hidrolización alcalina, usados principalmente en Estados Unidos y Europa. Además, enfatizan en que el mundo académico aún no ha despertado al potencial de estas dos nuevas tecnologías, debido a la escasez de artículos que aborden estos procesos; esta escasez puede deberse a que el sector funeral sigue siendo un área con poco interés por parte de los investigadores, en virtud de tratar a personas muertas, un asunto que muchos evitan.

\section{Transición del ritual funerario}

Pochintesta (2016), en su artículo proyecta las preferencias rituales en torno a la propia muerte, expresadas por personas de mediana edad: 40 a 60 años, y de cuarta edad: mayores de 80 años, reflejando en sus resultados una transformación de las prácticas y costumbres funerarias, en el contexto urbano de los últimos años. El autor concluye que, en la mediana edad, existe una preeminencia de la cremación que define la desmaterialización de la muerte y la apatía al cementerio y las ceremonias fúnebres; caso contrario a los de cuarta edad que tienen una valorización del cementerio y de la inhumación, estructurando una relación diferente entre vivos y muertos.

Duarte (2012), analiza la serie televisiva Seis palmos bajo tierra, 2000-2005, de Allan Ball, desde el punto de vista de diversos autores que consideran que la muerte en Estados Unidos es un negocio y el ritual funerario un evento que ha perdido su sentido, desplazando el proceso de duelo. Sin embargo, el autor explica como la casa funeraria Fisher y Sons ha dado importancia a este proceso, al tiempo que celebra la vida; de esta manera revela la importancia de la serie como un elemento visual que contribuye para la catarsis emocional del 
individuo y de la nación, principalmente en el caso de los ataques del 11 de septiembre en Estados Unidos.

La organización Memora, se destaca por estar conformada por 1.300 empleados y 130 empresas funerarias que le han permitido ganar el 13\% del mercado en España y el 5\% en Portugal, países en los que gestiona más de 120 tanatorios, 20 crematorios y 21 cementerios. Esta organización, en colaboración con la empresa IrisGem, ofrece el diamante IrisGem, el cual se da como un recuerdo obtenido a partir del carbono presente en las fibras capilares humanas. La Organización ha promovido el único máster especializado en temas funerarios, que se organiza en toda Europa: Funeral Services Master in Business Administration, Fusemba, programa que desarrolla en colaboración con el Instituto de Formación Continua de la Universidad de Barcelona, y con el soporte de la Comisión Europea (Carazo, 2012).

Beard y Burger (2017), describen los cambios generados entre el funeral "moderno" y el tradicional, debido al aumento de la riqueza en la sociedad estadounidense, que ha originado nuevos puntos de vista culturales sobre la muerte. Los autores indican que el ritual funerario tradicional, sigue siendo la selección funeraria más popular en los Estados Unidos. Sin embargo, se resalta que desde mediados del siglo XX y hasta ahora, el funeral moderno ha tomado gran fuerza en la industria. Los cambios van desde la adopción de una nueva metodología de embalsamamiento introducida en el siglo XIX, con cambios en esta práctica y la profesionalización del embalsamador, hasta tendencias recientes que implican un mayor uso de la tecnología y una variedad de formas alternativas para conmemorar a los muertos y deshacerse de los restos, que difieren del funeral tradicional.

\section{Alternativas ecológicas para la disposición de restos}

En la búsqueda de información, se encontraron trabajos de grado que proyectan nuevas alternativas frente a la disposición de restos, con el fin de minimizar el impacto ambiental. A continuación se mencionan los trabajos más relevantes.

Tecnologías alternas eco sustentables en el depósito de restos humanos Panteón civil San Lorenzo Tezonco, es una tesis de grado que busca generar una alternativa eco sustentable para la disposición de restos humanos, donde el nivel de ocupación sea controlado y armonioso con el entorno, generando un edificio con jardines verticales y azoteas verdes que promueven la eliminación de agentes contaminantes en el aire. Esta tesis permite identificar que el ritual funerario más común es la inhumación, donde el $80 \%$ opta por esta práctica, mientras que un $20 \%$ opta por la cremación (Bautista-Rodríguez, 2013).

López (2011), en su tesis: plan de negocios para una empresa productora y comercializadora de ataúdes fúnebres metálicos con sede en Bogotá," describe que la falta de cultura en el uso de materiales sustitutos y la resistencia al cambio de las funerarias, son las dos barreras de entrada más importantes para este tipo de empresas, limitando su campo de acción.

Ferreira (2016), proyecta la viabilidad de implementar prácticas alternativas para la eliminación de restos humanos en Portugal, enfatizando en las prácticas del entierro natural y la hidrólisis alcalina, tomando como base una perspectiva histórica, legal y de salud pública del entorno. El estudio conluye que la adopción de prácticas innovadoras de eliminación del cuerpo, como la hidrólisis alcalina, es muy poco probable en la actualidad, siendo el entierro natural el de mayor probabilidad en Portugal.

El Estudio para el diseño del cementerio ecológico municipal de la parroquia urbana del cantón Milagro provincia del Guayas, describe que la población de ese lugar ha experimentado un crecimiento demográfico, donde la ciudad de Milagro se ha convertido en el área de mayor concentración poblacional. Esto ha generado la necesidad de crear un nuevo cementerio, planteándose la idea que 
este tenga un enfoque ecológico, el cual tendrá en sus zonas de inhumaciones un sistema de fachadas verdes o muros vegetales que permiten reducir la velocidad del viento en contacto con la fachada, evitando su enfriamiento, a la vez queevitan el paso del agua de lluvias (Rivadeneira, 2016).

Feasibility study of a solar crematorium in india, citado en Kashyap y Kesari (2014), refiere que en el proceso de cremación se derriban muchos árboles para cumplir con este procedimiento, lo que contribuye significativamente en el calentamiento global y la contaminación el aire. Igualmente, se afirma que la religión hindú permite la cremación del cadáver únicamente durante el día, por lo cual un crematorio solar en este territorio es necesario. El horno crematorio busca encender el cadáver localmente en cualquier lugar, con la ayuda de un gran reflector concentrador que permita mantener la combustión del cadáver, mediante el suministro del aire atmosférico fresco con la ayuda de un soplador.

\subsection{Búsqueda en bases de datos}

Para identificar invenciones relacionadas con el factor crítico de vigilancia, se realizó una búsqueda a través de la plataforma Google Patents. La información de las patentes relevante se presenta a continuación, en las tablas 4 a 15.

Tabla 4. Eliminación de restos humanos (Sullivan, 2013).

\begin{tabular}{ll}
\hline Resumen & Características de la Patente \\
\hline $\begin{array}{l}\text { La patente describe un ataúd para el uso en procesos de hidrólisis alcali- } \\
\text { na, el cual comprende un receptáculo soluble y disoluble en el proceso; }\end{array}$ & Registrada/2008 \\
también se describe un método para deshacerse de un cadáver. & Publicada: el 3/06/2013 \\
& Número de publicación: ES2405756T3A \\
& Inventor: Alexander Sullivan \\
\hline
\end{tabular}

Tabla 5. Procedimiento para la obtención de un producto sólido a base de cenizas, CAMPS (Quim, 2016).

Resumen Características de la Patente

La invención divulga un procedimiento para la obtención de un producto Registrada: 4/07/2014 sólido con cenizas de cremación de un humano o animal, en el interior de un recipiente

Publicada: 08/01/2016

Número de publicación: ES2555829B1

Inventor CAMPS, Quim 
Tabla 6. Procedimiento para obtener materiales cerámicos a partir de cenizas de seres queridos (Casañi, 2011).

\section{Resumen}

Procedimiento para obtener materiales cerámicos a partir de cenizas de seres queridos, consistente en que una vez recibidas las cenizas procedentes

de un proceso de cremación con la certificación de identificación, son so- Publicada: el 3/05/2011 metidas a 7 fases

\section{Características de la Patente}

Registrada: 19/09/2008

Número de publicación: ES2342353B1

Inventor: Esther Casañi Giner

Tabla 7. Construcción de contención del cuerpo adecuada para usar en procesos de bio-cremación (Frenton \& Lefevre, 2018).

Resumen

Las construcciones de contención de la patente, proporcionan la funcionalidad de contención de líquido y también la funcionalidad de la presentación del cuerpo para fines de visualización, si se desea, además de la biodegradabilidad.

\section{Características de la Patente}

Registrada: 07/03/2017

Publicada: 9/01/2018

Número de publicación: US9861546B2

Inventor: Renata Fenton Anne Sofie Lefèvre

Tabla 8. Urna biodegradable (Moline, 2006).

Resumen

Características de la Patente

La invención describe una urna biodegradable de aplicaciones funerarias, Registrada: 31/05/2005 que alberga en su interior unas cenizas producto de la incineración de un difunto y al menos una semilla de una planta, que cuenta con un recipiente Publicada: 16/05/2006 de material celulósico en cuyo interior se alojan las cenizas.

Número de publicación: ES1060383U

Inventor: Gerard Moline Navarro

Tabla 9. Ataúd ecológico (Fernadiz, 2012)

Resumen Características de la Patente

Se describe un ataúd ecológico constituido en cartón biodegra- Registrada: 02/12/2011 dable y reciclado, comprendido de una caja y una tapa, ambas constituidas por piezas laminares de cartón debidamente tro- Publicada: 30/03/2012 queladas para su armado.

Número de publicación: ES1075932U

Inventor: Francisco Javier Ferrándiz Moreno 
Tabla 10. Sistema para termofusionar cenizas de seres vivos entre vidrios a alta temperatura (Ares, 2012).

\begin{tabular}{|c|c|}
\hline Resumen & Características de la Patente \\
\hline $\begin{array}{l}\text { Esta invención busca incluir entre dos o más piezas de cualquier tipo de vidrio } \\
\text { existente en el mercado, las cenizas resultantes de la cremación de seres vivos, } \\
\text { (personas o animales) y someter esta combinación a temperaturas superiores } \\
\text { a } 800 \text { grados por medios industriales, para realizar esculturas, colgantes o cual- } \\
\text { quier objeto de este tipo. }\end{array}$ & $\begin{array}{ll}\text { de vidrio } & \text { Registrada: 11/07/2012 } \\
\text { res vivos, } & \\
\text { uperiores } & \text { Publicada: 15/01/2014 } \\
\text { es o cual- } & \\
& \text { Número de publicación: } \\
& \text { ES2438004A1 } \\
& \text { Inventor: Julia Ares Iglesias }\end{array}$ \\
\hline Resumen & Características de la Patente \\
\hline $\begin{array}{l}\text { La invención describe un ataúd de material respetuoso con el medio } \\
\text { ambiente con una forma especial y accesorios en los lugares por los } \\
\text { que va a levantarse el ataúd, en el que el ataúd en sí mismo tiene } \\
\text { rebajes verticales dirigidos hacia dentro, que abarcan tanto la caja } \\
\text { como la tapa del ataúd }\end{array}$ & $\begin{array}{l}\text { Registrada: 29/04/2011 } \\
\text { Publicada: 29/07/2015 } \\
\text { Número de publicación: ES2541406T3 } \\
\text { Inventor: Bendt Skov, Jakob Brahe Pedersen, } \\
\text { Nils Thobo Carlsen }\end{array}$ \\
\hline
\end{tabular}

Tabla 12. Urna funeraria y método para su elaboración (Álvarez \& Carrillo, 2013).

Resumen Características de la Patente

La urna funeraria está conformada por una mezcla compac- Registrada: 30/09/2011 tada de granos o partículas, constituidas principalmente por sal y opcionalmente otros aditivos, siendo estos aditivos Publicada: 21/05/2013 unos colorantes o aromas.

Número de publicación: ES2403739B1

Inventor: Dolores Álvarez del Castillo, Fernando Carrillo Navarrete

Tabla 13. Estructura memorial para urnas cinerarias (Bach, 2014).

\begin{tabular}{ll}
\hline Resumen & Características de la Patente \\
\hline La invención está constituida por un bloque envolvente que aloja varios co-- & Registrada: 11/07/2012 \\
$\begin{array}{l}\text { lumbarios que delimitan espacios para alojamiento de las urnas cinerarias, } \\
\text { cerrados por las tapas individuales, sobre las que se fijan una pluralidad de }\end{array}$ & $\begin{array}{l}\text { Publicada: 20/02/2014 } \\
\text { piezas frontales de cerámica, piedra natural o artificial, plástico y similares. }\end{array}$ \\
& Número de publicación: ES2443867B1 \\
& Inventor: Santiago Bach Lahor \\
\hline
\end{tabular}


Tabla 14. Método y aparato para tratar restos humanos mediante refrigeración (ECOLEGACY Ltd, 2015).

\begin{tabular}{ll}
\hline Resumen & Características de la Patente \\
\hline $\begin{array}{l}\text { La invención describe el método para el tratamiento de la materia orgánica } \\
\text { para su descomposición después de la criodesecación, en el que el material }\end{array}$ & Registrada: 04/12/2000 \\
$\begin{array}{l}\text { orgánico en el estado de congelación, está sujeto al proceso de despiece y } \\
\text { el material está entonces sujeto al proceso de criodesecación antes de ser }\end{array}$ & Publicada: 16/05/2005 \\
trasladado para su eliminación mediante descomposición. & Número de publicación: \\
& US20150067998A1 \\
& $\begin{array}{l}\text { Inventor: Damian Tinsley, Joe Ennis, } \\
\text { Stephen Lusty }\end{array}$ \\
\hline
\end{tabular}

Tabla 15. Panteón Botánico (Sepúlveda, 2011).

Resumen Características de la Patente

Está enfocada en la inhumación ecológica, a partir del proceso de enterrar Registrada: 21/12/2010 el cuerpo sin vida de una persona al pie de un árbol dentro de un ataúd biodegradable para que, con la biodegradación natural, los restos se reintegren a la naturaleza.

Publicada: 01/12/2011

Nmero de publicación: WO2011078640A2

Inventor: Fernando Sepúlveda

\subsection{Mapa tecnológico con análisis de frecuencias}

En esta fase se seleccionaron artículos que fuesen pertinentes a la tendencia tecnológica, en cuanto a las prácticas funerarias para el destino final. Una vez identificadas las palabras clave de cada artículo, se realizó una búsqueda en las bases de datos: Enviroment Complete, Fuente Académica, Med- line, Science Direct, Dialnet y Google patents; en ellas se encontraron artículos e invenciones pertinentes a estas palabras clave, relacionadas, directa o indirectamente, con el factor crítico a vigilar. Lo anterior permitió identificar la cantidad de artículos y patentes publicadas, realizando un análisis de coocurrencia como se ilustra en la figura 2 , junto con un mapa tecnológico descrito en la figura 3.

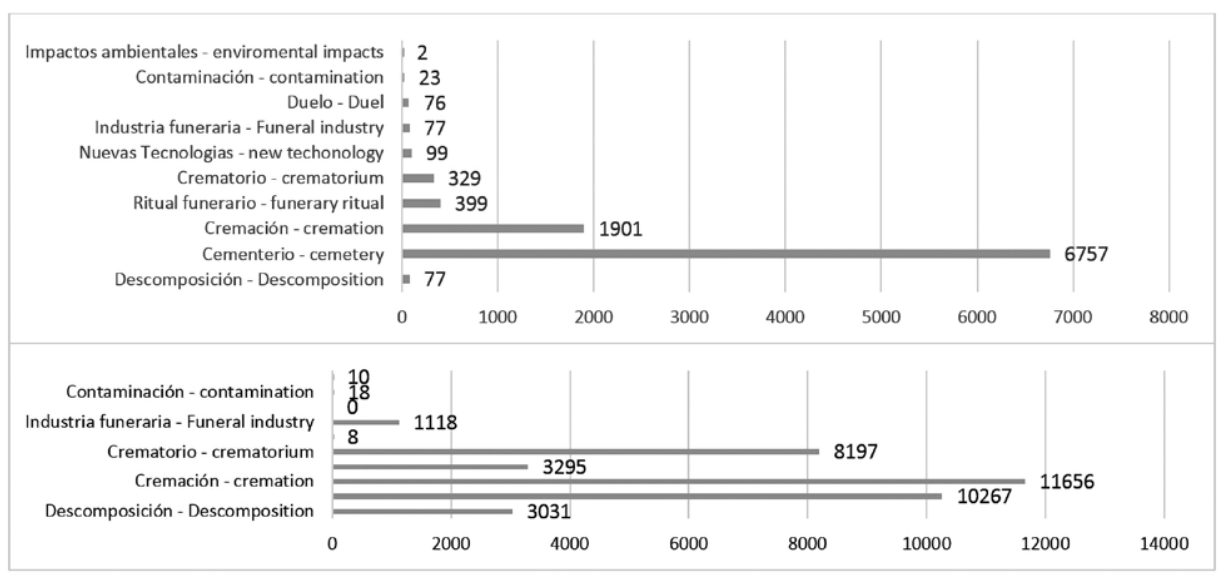

Figura 2. Análisis de coocurrencia para artículos científicos y patentes. 


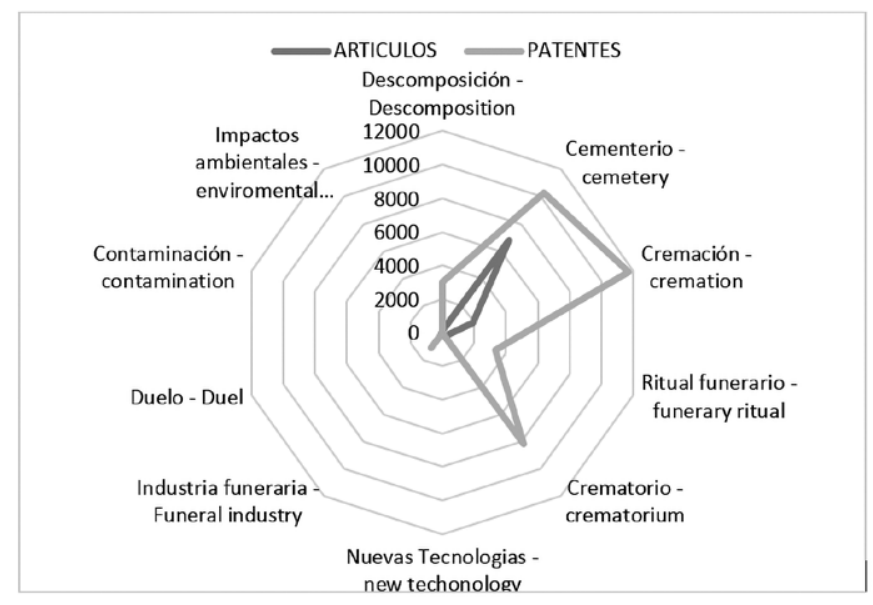

Figura 3. Relación de resultados entre artículos científicos y patentes.

En el mapa de relación de resultados de artículos científicos y patentes de la figura 3, se observa que existe un mayor índice de invención que de producción académico científica. Las palabras clave: cementerio, cremación y crematorio, tienen mayor incidencia en las patentes; mientras que cementerio es la palabra clave más frecuente en la producción científica.

\section{Conclusiones}

Existe una laguna de información importante en la temática del sector funerario, tanto en Colombia como a nivel internacional, lo cual dificultó el rastreo de información para el desarrollo del proceso de vigilancia tecnológica. Es notorio que a nivel de pregrado se han adelantado varios proyectos, que abarcan de una u otra forma diversos aspectos del sector estudiado.

El mapa tecnológico permitió identificar que es mayor la generación de patentes que la publicación de artículos. Se evidenció que el sector funerario tiene patentes para diferentes procesos que emergen de esta actividad económica. En este sentido, la empresa Grupo Obelisco Ltda. podrá generar vigilancia tecnológica en diferentes aspectos, según las necesidades actuales o futuras de la organización.
Pese a que Colombia es un país con proyección a nivel internacional en innovación del sector funerario, lo que le ha permitido un crecimiento importante en los últimos años, se evidencia la poca participación en cuanto a producción bibliográfica y nula participación en desarrollo de patentes, referentes al destino final de los restos humanos. Esto establece la necesidad que tienen las empresas del sector, en cuanto a fortalecer la creación de departamentos de innovación y desarrollo que les permita estar a la vanguardia y salvaguardar sus posibles invenciones.

Teniendo en cuenta las características de la empresa bajo estudio, los hallazgos permiten concluir que las tendencias tecnológicas que pueden ser consideradas por la organización para ampliar su portafolio, con la implementación de destino final, son los procesos de: hidrólisis alcalina, criodesecación, además del cementerio ecológico y la urna biodegradable. Estos procesos, en conjunto, generarían una cadena de valor diferenciadora para la empresa en el mercado regional, departamental y nacional.

Finalmente, ante la decisión que tome la empresa Grupo Obelisco frente a la diversificación de su portafolio de servicios con la inclusión de la opción de destino final, se debe generar una investigación de mercados que le permita conocer la 
percepción de sus clientes actuales y potenciales, teniendo en cuenta las características socioculturales del departamento de Boyacá.

\section{Agradecimientos}

La autora agradece a la Universidad Nacional Abierta y a Distancia, UNAD, donde adelantó los estudios de Maestría en Administración de Organizaciones, desarrollando el proyecto aquí reportado.

\section{Referencias}

Ahumada-Tello, E., Zarate-Cornejo, R. E., Plascencia-López, I., \& Perusquia-Velasco, J. M. A. (2012). Productivity Model Based on Knowledge-The Case of the Information Technology Pymes in Baja California. Revista Internacional Administración y Finanzas, 5 (4), 13-27. Recuperado de: http://www. theibfr2.com/RePEc/ibf/riafin/riaf-v5n4-2012/ RIAF-V5N4-2012-2.pdf

Ares, J. (2012). Sistema para termofusionar cenizas de seres vivos entre vidrios a alta temperatura. ES2438004A1

Álvarez, D, \& Carrillo, F. (2013). Urna funeraria y método para su elaboración. ES2403739B1

Bach, S. (2014). Estructura memorial para urnas cinerarias. ES2443867B1

Bautista-Rodríguez, I. M., (2013). Tecnologías alternas eco sustentables en el depósito de restos humanos Panteón civil San Lorenzo Tezonco (tesis pregrado). Escuela Superior de Ingeniería y Arquitectura, México.

Beard, V. R., \& Burger, W. C. (2017). Change and innovation in the funeral industry: A typology of motivations. OMEGA-Journal of Death and Dying, 75 (1), 47-68. doi: https://doi. org/10.1177/0030222815612605
Carazo, J. (2012). Memora lidera la profesionalización del sector funerario. Revista Capital Humano, 25 (261), 42-50.

CAMPS, Quim (2016). Procedimiento para la obtención de un producto sólido que comprende cenizas de cremación y una cera en un recipiente, producto obtenido mediante el procedimiento y estuche que contiene dicho producto. ES2555829B1.

Casañi, E. (2011). Procedimiento para obtener materiales cerámicos a partir de cenizas de seres queridos. ES2342353B1

Da Cruz, N. J. T., Lezana, Á. G. R., Dos Santos, P. D. C. F., Zancan, C., \& Pinto, I. M. B. S. (2015). Cemitérios, crematórios e novas tecnologias fúnebres: impactos ambientais e preferências post-mortem na cidade de Maceió-AL. Revista Eletronica Gestão y Saúde, 1 (1), 1058-1072. Recuperado de: http://periodicos.unb.br/index.php/rgs/article/ view/22617/16167

Duarte, J. (2012). Resting in peace: the work of mourning in Six Feet Under by Allan Ball. Cuadernos de Música, Artes Visuales y Artes Escénicas, 7 (1), 133-146. Recuperado de: http://www.redalyc.org/ pdf/2970/297023537006.pdf

ECOLEGACY Ltd. (2015). Método y aparato para tratar restos humanos mediante refrigeración. US20150067998A1

Fenton, R., \& Lefevre, A (2018). Construcción de contención del cuerpo adecuada para usar en procesos de bio-cremación. US9861546B2

Ferreira, A. R. (2016). Body disposal in Portugal: Current practices and potential adoption of alkaline hydrolysis and natural burial as sustainable alternatives (tesís de maestría). Universidade do Porto, Portugal

Ferrandiz, F. (2012). Ataúd ecológico. ES1075932U 
Gaioli, M., Amoedo, D., \& González, D. (2012). Impacto del mercurio sobre la salud humana y el ambiente. Archivos argentinos de pediatría, 110 (3), 259-264. Recuperado de: https://www.researchgate.net/publication/272990424_Impacto_del_mercurio_sobre_la_salud_humana_y_el_ambiente

González, A. I., Gómez, D., \& Muñoz, L. (2015). Guía práctica Innovitech. Vigilancia Tecnológica para la Innovación. Recuperado de http://www.ovtt.org/ sites/default/files/archivos/Gu\%C3\%ADa\%20Pr\%C3\%A1ctica\%20I nnoViTech\%202015.

Kashyap, A. C., \& Kesari, J. P. (2014). Feasibility Study of a Solar Crematorium in India. I, (IV), 1-9. Recuperado de: https://www.rsisinternational. org/lssue4/01-09.pdf

López, Á. (2011). Plan de negocios para una empresa productora y comercializadora de ataúdes fúnebres metálicos con sede en la ciudad de Bogotá (tesis de pregrado). Pontificia Universidad Javeriana, Bogotá, Colombia.

Marulanda, C. E., Hernández, A., \& López, M. (2016). Vigilancia Tecnológica para Estudiantes Universitarios: El Caso de la Universidad Nacional de Colombia, Sede Manizales. Formación universitaria, 9 (2), 17-28. Recuperado de: https://scielo. conicyt.cl/pdf/formuniv/v9n2/art03.pdf

Mari, M., \& Domingo, J. L. (2010). Toxic emissions from crematories: a review. Environment international, 36 (1), 131-137. Recuperado de: https:// www.semanticscholar.org/paper/Toxic-emissions-from-crematories\%3A-a-review.-Mari-Domingo/3af1c4a451b176e31341b7429b5ccb55dc568a87

Moline, G (2006). Urna biodegradable. ES1060383U

Nonaka, I., Takeuchi, H., \& Kocka, M. H. (1999). La organización creadora de conocimiento: cómo las compañías japonesas crean la dinámica de la innovación, 61-103. México DF: Oxford University Press
Pochintesta, P. A. (2016). La ritualidad en transición. Un estudio sobre las preferencias del destino corporal. Athenea Digital. Revista de pensamiento e investigación social, 16 (2), 33-66. Recuperado de: http://atheneadigital.net/article/view/v16-n2-pochintesta/1559-pdf-es

Quintero-Castillo, L. C. (2017). Análisis de la gestión de riesgos en laboratorios de tanatopraxia según lineamientos metodológicos de la guía de fundamentos para la dirección de proyectos (PMBOK) (tesis de grado). Universidad Militar Nueva Granada.

Rivadeneira-Pino, M. D. L. A. (2016). Estudio para el diseño del cementerio ecológico municipal para la parroquia urbana del cantón Milagro provincia del Guayas (tesis pregrado). Universidad de Guayaquil, Guayaquil, Ecuador

Recondo-Pérez, R. F. (2013). La Arquitectura del Crematorio. Función, estética y medio ambiente. Revista de Arquitectura e Ingeniería, 7 (2), 1-26. Recuperado de: http://www.redalyc.org/articulo. oa?id=193929227002

Rodríguez-Parra, R. del M. (2018). Estudio de vigilancia tecnológica e inteligencia competitiva para la empresa Grupo Obelisco Ltda. Orientado a la diversificación de su portafolio de servicios en la implementación del proceso de destino final (Tesis de maestría). Universidad Nacional Abierta y a Distancia, Sogamoso, Colombia

Rodríguez-Gómez, D. (2006). Modelos para la creación y gestión del conocimiento: una aproximación teórica. Educar, 37, 25-39. Recuperado de: https://ddd.uab.cat/pub/educar/0211819Xn37/0211819Xn37p25.pdf

San Juan, Y. I., \& Romero-Rodríguez, F. I. (2016). Modelos y herramientas para la vigilancia tecnológica. Ciencias de la Información, 47 (2), 1118. Recuperado de: http://www.redalyc.org/ html/1814/181452083002/ 
Sepúlveda, F. (2011). Panteón botánico. Velasco-Rivera, A., Zea, M., \& Marlevis, Y. (2012). WO2011078640A2

Smith, T. O., Gitsham, P., Donell, S. T., Rose, D., \& Hing, C. B. (2012). The potential dangers of medical devices with current cremation practices. European Geriatric Medicine, 3 (2), 97-102. Recuperado de: https://www.researchgate.net/publication/257730153_The_potential_dangers_of_medical_devices_with_current_cremation_practices

Skov, B., Brahe, J., \& Thobo, N. (2015). Ataúd fabricado de material respetuoso con el medio ambiente. ES2541406T3

Sullivan, A. (2013). Eliminación de restos humanos. ES2405756T3 Assessment by Soil Pollution Bordering Burial Grounds Jardines Del Recuerdo And Inmaculada. Ciencia e Ingeniería Neogranadina, 22 (1), 165-175. Recuperado de: http://www. scielo.org.co/scielo.php?script=sci_arttext\&pi$d=$ S0124-81702012000100011

Xue, Y., Tian, H., Yan, J., Xiong, C., Pan, T., Nie, L., \& Zhu, C. (2016). Present and future emissions of HAPs from crematories in China. Atmospheric Environment, 124, 28-36. Recuperado de: https://www. researchgate.net/publication/284086922_Present_and_Future_Emissions_of_HAPs_from_Crematories_in_China 